\title{
Activating transcription factor 2 expression mediates cell proliferation and is associated with poor prognosis in human non-small cell lung carcinoma
}

\author{
ZHENYU YOU ${ }^{1}$, YONG ZHOU ${ }^{2}$, YULING GUO ${ }^{1}$, WENYAN CHEN ${ }^{1}$, SHAOQING CHEN ${ }^{1}$ and XIAOLANG WANG ${ }^{1}$ \\ ${ }^{1}$ Department of Oncology, First Affiliated Hospital of Nanchang University, Nanchang, Jiangxi 330006; \\ ${ }^{2}$ Department of Pharmacy, Peking University, Beijing 100083, P.R. China
}

Received October 1, 2014; Accepted August 20, 2015

DOI: $10.3892 / \mathrm{ol} .2015 .3922$

\begin{abstract}
Activating transcription factor 2 (ATF2) is a member of the cAMP response element binding protein family that heterodimerizes and activates other transcription factors involved in stress and DNA damage responses, growth, differentiation and apoptosis. ATF2 has been investigated as a potential carcinogenic biomarker in certain types of cancer, such as melanoma. However, its function and clinical significance in non-small cell lung cancer (NSCLC) has not been well studied Therefore, the present study aimed to analyze the association between ATF2/phosphorylated (p)-ATF2 expression and NSCLC malignant behavior, and discuss its clinical significance. Reverse transcription-quantitative polymerase chain reaction and western blotting were used to detect the expression of ATF2 in NSCLC cell lines and fresh NSCLC tissue samples. In addition, immunohistochemistry (IHC) was performed to identify the location and expression of ATF2 and p-ATF2 (threonine 71) in paraffin-embedded sections of NSCLC and adjacent normal tissue. The results demonstrated that ATF2 was markedly overexpressed in the NSCLC cells and significantly overexpressed in the fresh NSCLC tissues compared with the control cells and samples (86 paraffinembedded tissue sections), respectively $(\mathrm{P}<0.01)$. Further data demonstrated that ATF2 expression levels were significantly increased in tumor tissues compared to normal tissues and ATF2 was located in the cytoplasm and nucleus. ATF2 expression was closely associated with adverse clinical characteristics such as TNM stage $(\mathrm{P}=0.002)$, tumor size $(\mathrm{P}=0.018)$ and metastasis $(\mathrm{P}=0.027)$. In addition, nuclear $\mathrm{p}-\mathrm{ATF} 2$ staining was positive in 65/86 samples of NSCLC. Furthermore, the Kaplan-Meier analysis indicated that patients with high levels of ATF2 and
\end{abstract}

Correspondence to: Professor Xiaolang Wang, Department of Oncology, First Affiliated Hospital of Nanchang University, 17 Yongwai Street, East Lake, Nanchang, Jiangxi 330006, P.R. China E-mail: wangxiaolangmed@163.com

Key words: activating transcription factor 2, non-small cell lung carcinoma, proliferation, clinicopathology, prognosis
p-ATF2 expression had a significantly shorter overall survival compared with patients exhibiting a low expression $(\mathrm{P}<0.01$ and $\mathrm{P}<0.05$, respectively). Subsequent in vitro experiments revealed that cell growth decreased following knockdown of ATF2 expression using RNA interference, indicating that ATF2 may suppress cell proliferation. Taken together, the results of the present study demonstrated that ATF2 and p-ATF2 were significantly overexpressed in NSCLC tissues, and ATF2 and p-ATF2 overexpression predicted significantly worse outcomes for patients with NSCLC.

\section{Introduction}

Lung cancer is characterized by the highest incidence and mortality rates of any malignant tumor (1), and non-small cell lung cancer (NSCLC) accounts for $\sim 85 \%$ of all the diagnostic cases of lung cancer (2). For decades, numerous therapeutic strategies, including adjuvant, neoadjuvant and postoperative chemotherapy, have been used in the treatment of NSCLC (3). Recently, epidermal growth factor receptor tyrosine kinase inhibitors, including gefitinib, erlotinib and afatinib, have been used in the treatment of NSCLC, resulting in lower toxicity and higher efficiency, compared with traditional chemotherapeutic drugs (4). However, patients with NSCLC still failed this novel chemotherapy regime, and exhibited worse overall survival (OS) due to multidrug resistance and the heterogeneity of NSCLC tumors (5). It currently remains difficult to obtain an early diagnosis and execute timely therapeutic interventions for patients with NSCLC; therefore, novel effective therapeutic strategies should be investigated (6). Furthermore, novel biological markers for the early diagnosis and survival prediction of NSCLC are emerging.

Activating transcription factor 2 (ATF2), a member of the cAMP response element binding family, is activated by phosphorylation to c-Jun N-terminal kinases or p38, and heterodimerizes with those transcription factors in response to regulatory biological progresses $(7,8)$, such as stress and cytokines (9-11). ATF2 dimerizes with other members of the activator protein 1 superfamily to activate the transcription of genes involved in stress and DNA damage responses, growth, differentiation, and apoptosis (9-11). Furthermore, ATF2 can be activated by stress and cytokine stimuli in response to a 
variety of cell processes, including DNA damage, viral infection and cell death (9). It has previously been demonstrated that ATF2 has an oncogenic role in melanocyte transformation (12). Notably, ATF2 executes different roles depending on its localization. For example, the nuclear localization has been demonstrated to be associated with poor prognosis in patients with melanoma, suggesting that ATF2 localization may serve as a prognostic marker in this disease (13). In addition, protein kinase $\mathrm{C} \varepsilon(\mathrm{PKC} \varepsilon$ ) may phosphorylate ATF2 on threonine 52 and localize ATF2 to the nucleus (13). Elevated levels of $\mathrm{PKC} \varepsilon$, which are observed in more advanced stage metastatic melanomas, appear to prevent the nuclear-to-mitochondrial translocation of ATF2 that enable its tumor-suppressor function (14), and ATF2 targets include various pro-survival molecules, such as cell cycle-related proteins (15). Furthermore, a previous study indicated that enhancing the nuclear export of ATF2 may be a novel therapeutic modality for drug-resistant melanomas (5).

In the present study, the expression and localization of ATF2 was detected in NSCLC cancer tissues and cells. Subsequently, the association between ATF2 expression and clinicopathological characteristics were analyzed to determine the clinical significance of ATF2 in the prognostic prediction of patients with NSCLC. The results demonstrated that the expression levels of ATF2 and p-ATF2 were markedly increased in NSCLC tissues. ATF2 is therefore another promising predictor for prognosis of NSCLC.

\section{Materials and methods}

Cell culture. Normal human bronchial epithelial (HBE) cells (HBE 135-E6E7) and various human NSCLC cells (H1299, H1975, A549, PC9, H460 and H1650) were purchased from the American Type Culture Collection (Manassas, VA, USA). The H1299, H1975, H460 and H1650 cells were cultured in RPMI-1640 and the PC9 and A549 cells were cultured in Dulbecco's modified Eagle's medium (ThermoFisher Scientific, Inc., Waltham, MA, USA). The culture medium contained $10 \%$ heat-inactivated fetal bovine serum (FBS; ThermoFisher Scientific, Inc.) and antibiotics (100 U/ml penicillin and $100 \mathrm{U} / \mathrm{ml}$ streptomycin; Beyotime Institute of Biotechnology, Inc., Dalian, China). HBE cells were cultured in RPMI-1640 with $15 \%$ FBS. All the cells were incubated at $37^{\circ} \mathrm{C}$ and $5 \% \mathrm{CO}_{2}$ in a humidified atmosphere.

Patients and tissue specimens. Written informed consent was obtained fromall patients.Paired cancerous and adjacent normal tissue samples were obtained from 86 patients with NSCLC who underwent surgical resection at the First Affiliated Hospital of Nanchang University (Nanchang, China) between June 2001 and July 2004, according to protocols approved by the Institute Research Medical Ethics Committee of the First Affiliated Hospital of Nanchang University. The 86 patients were aged between 24 and 68 years (median age, 42 years), and the tumors were pathologically graded according to the International Union against Cancer tumor-node-metastasis classification of malignant tumors. Following surgical resection, the 86 tissues specimens were immediately fixed in $10 \%$ formalin, embedded in paraffin and stored at room temperature. In addition, to investigate RNA levels of AFT2 in fresh tumor tissues, 12 paired fresh tumor and adjacent normal tissues were obtained from NSCLC patients who underwent surgical resection at the First Affiliated Hospital of Nanchang University between November 2011 and February 2012. The 12 patients were aged between 41 and 60 years (median age, t51.5 years). Following surgical resection, the 12 paired tumor and adjacent normal tissue specimens were immediately frozen in RNAlater ${ }^{\circledR}$ (Ambion Life Technologies, Carlsbad, CA, USA) and stored at $-80^{\circ} \mathrm{C}$.

Immunohistochemistry (IHC). The tissues were fixed with 4\% paraformaldehyde in $0.1 \mathrm{M}$ phosphate buffer. After $24 \mathrm{~h}$, the tissues were embedded in paraffin wax and sliced into $14 \mu \mathrm{M}$ sections and mounted onto glass slides. The paraffin-embedded tissue sections were then dewaxed in xylene and alcohol, boiled with EDTA ( $1 \mathrm{mmol} / \mathrm{l}$; $\mathrm{pH} 8.0$ ), and treated with $3 \% \mathrm{H}_{2} \mathrm{O}_{2}$ for $10 \mathrm{~min}$. Immunohistochemical analysis was then performed using the EnVision ${ }^{\mathrm{TM}}$ two-step staining method (16). Briefly, the slides were incubated with primary polyclonal rabbit anti-human ATF2 (1:500 dilution; p-ATF2, 1:1,000 dilution; Santa-Cruz Biotechnology, Inc.) or mosue anti-human GAPDH (1:1,000 dilution; Zhejiang Kangchen Biotech Co., Hangzhou, China) overnight at $4^{\circ} \mathrm{C}$ and then washed in phosphate-buffered saline three times before the goat anti-rabbit and anti-mouse IgG secondary antibodies (1:5,000 dilution; Zhejiang Kangchen Biotech Co.) were added. After $1 \mathrm{~h}$ at room temperature, the slides were stained with DAB and counterstained with Mayer's hematoxylin (Beyorime Institute of Biotechnology, Inc.). Slides that were not incubated with primary antibody were used as the negative controls. The proportion of stained cells was scored as previously described (17), and the receptor score was calculated using the equation: Positively stained cell proportion $\mathrm{x}$ staining intensity. The median value of the IHC scores was 4; therefore, low and high expression were set at scores of $<4$ and $\geq 4$, respectively (18).

$R N A$ extraction and reverse transcription-quantitative polymerase chain reaction $(R T-q P C R)$. Total RNA was extracted from the 12 frozen samples using TRIzol ${ }^{\circledR}$ reagent (Bioo Scientific, Austin, TX, USA), according to manufacturer's instructions. RT-qPCR were performed using SYBR ${ }^{\circledR}$ Green Real-Time PCR Master Mix and the Stratagene Mx3000P Real-Time PCR system (Agilent Technologies, Inc., Santa Clara, CA, USA). The primer sequences were as follows: ATF2, sense 5'-GTAATCACCCAGGCACCATC-3' and antisense 5'-CTGAGTCCTAACCAATCCCC-3'; GAPDH, sense 5'-GAAGGTGAAGGTCGGAGTC-3' and antisense 5'-GAAGATGGTGATGGGATTTC-3'. The cycling conditions were set as follows: $95^{\circ} \mathrm{C}$ for $10 \mathrm{~min}$ followed by 40 amplification cycles at $95^{\circ} \mathrm{C}$ for $10 \mathrm{sec}$ and $60^{\circ} \mathrm{C}$ for $20 \mathrm{sec}$. The GAPDH gene served as the control and relative mRNA expression levels were defined using the $2^{-\Delta \Delta \mathrm{Ct}}$ method (19).

Plasmids and short hairpin (sh)RNAs. shRNA probes targeting the ATF2 gene were purchased from Genechem, Inc. (Shanghai, China). The ATF2 gene sequence was 5'-GTT GGCGAGTCCATTTGAG-3' and transfection of H1299 and H1975 cells was performed using Lipofectamine 2000 (Invitrogen Life Technologies, Carlsbad, CA, USA), according to the manufacturer's instructions. The pGIPZ vector was 


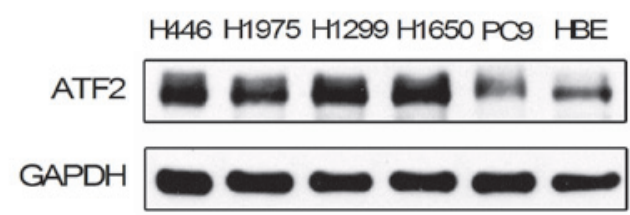

$\mathbf{C}$

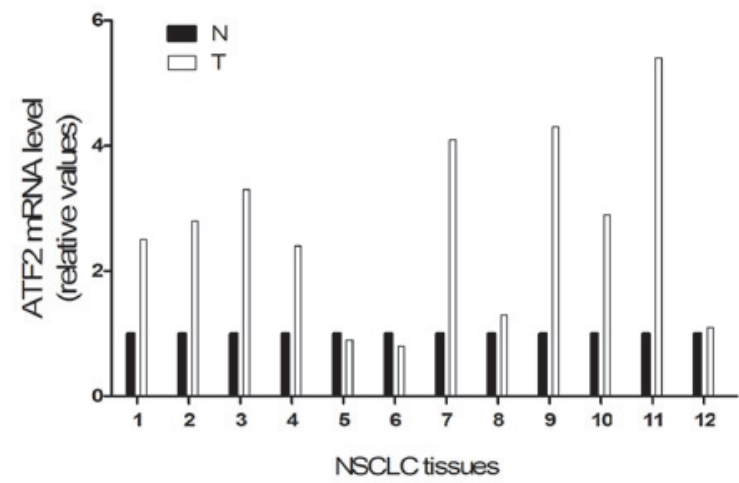

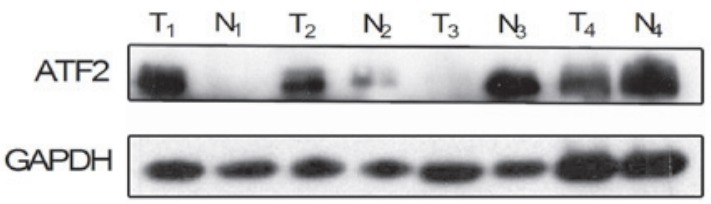

D

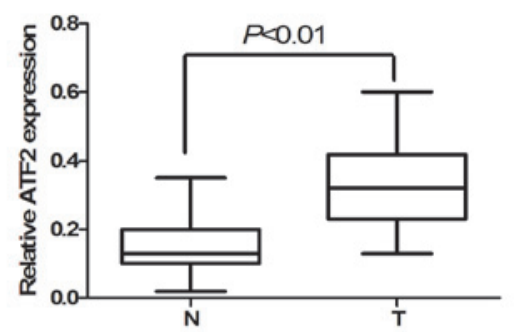

Figure 1. Expression of ATF2 in NSCLC tissue samples by western blotting and reverse transcription-quantitative polymerase chain reaction. Western blot analysis of ATF2 protein expression levels in (A) NSCLC cell lines and HBE cells and in (B) NSCLC and corresponding adjacent normal lung tissues, 4 samples are presented as being representative of the data. (C) Relative ATF2 mRNA expression levels in 12 pairs of NSCLC and corresponding adjacent normal lung samples. (D) A significant difference in ATF2 mRNA expression in normal (N) and tumor (T) tissues revealed by a Wilcoxon matched-pairs test. ATF2, activating transcription factor 2; NSCLC, non-small cell lung cancer.

purchased from Genechem, Inc. (Shanghai, China). Untransfected parental cells H1299 and H1975 were used as control. After $48 \mathrm{~h}$, the expression level of ATF2 was measured by western blotting and MTT assays.

Western blot analysis. Western blot analysis was performed to assess the protein expression levels of ATF2 and GAPDH in NSCLC cells and tissues, according to previously described protocols (20). The GAPDH gene served as the control. Briefly, The cells and tissues were lysed with lysis buffer ( $\mathrm{pH} 7.4$, containing $1 \%$ Triton X-100 and $0.2 \%$ SDS) on ice for $30 \mathrm{~min}$ and centrifugated at $10,000 \mathrm{rpm}$ for $15 \mathrm{~min}$ at $4^{\circ} \mathrm{C}$. The supernatant was separated and used for the experiment. The concentrations of protein were quantified using a bicinchoninic acid assay kit (ThermoFisher Scientific). Subsequently, $30 \mu \mathrm{g}$ protein from each sample were examined on $10 \%$ SDS-PAGE gels (Sigma-Aldrich). The separated proteins were transferred onto polyvinylidene fluoride membranes (EMD Millipore, Billerica, MA, USA) at $80 \mathrm{~V}$ for $100 \mathrm{~min}$ prior to blocking with $5 \%$ non-fat milk for $1 \mathrm{~h}$ at room temperature. The membranes were then incubated with primary antibodies against ATF2 (1:1,000 dilution) or GAPDH (1:1,000 dilution) overnight at $4^{\circ} \mathrm{C}$. After washing with Tris-buffered saline with Tween 20 three times, the membranes were incubated with peroxidase-conjugated secondary antibody (1:5,000 dilution) for $1 \mathrm{~h}$ at room temperature. The signal was then detected using an enhanced chemiluminescence detection system (GE Healthcare, Princeton, NJ, USA). The bands were exposed to the Kodak medical X-ray processor (Kodak, Rochester, NY, USA).

MTT assay. Cell viability was determined by performing an MTT assay (Sigma-Aldrich). Briefly, the cells were seeded at
A

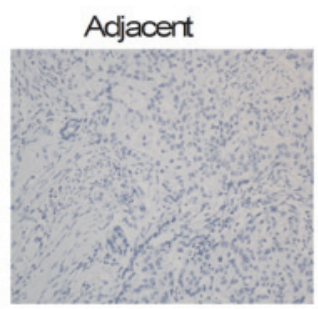

B

p-ATF2

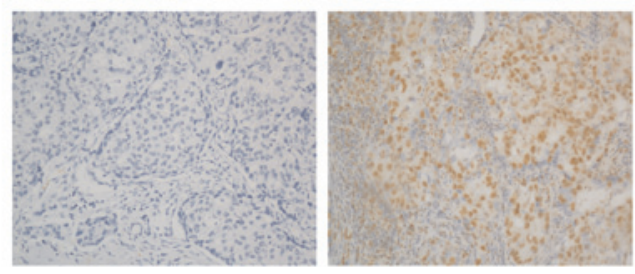

C

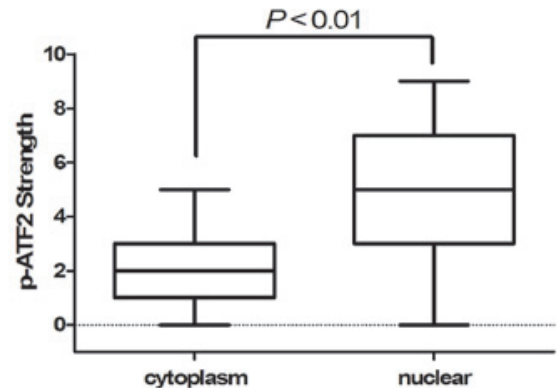

Figure 2. Expression of ATF2 in non-small cell lung cancer tissues by immunohistochemistry. Micrographs revealing (A) ATF2 and (B) p-ATF2 staining in normal adjacent lung and tumor tissues (DAB and Mayer's hematoxylin staining). (C) The strength and distribution of p-ATF2 in all 86 patients, calculated using the Wilcoxon matched-pairs test. p-ATF, phosphorylated activating transcription factor 2 . 
Table I. Correlation between ATF2 expression and clinicopathological parameters in non-small cell lung cancer.

\begin{tabular}{|c|c|c|c|c|}
\hline \multirow[b]{2}{*}{ Variable } & \multirow[b]{2}{*}{ Total cases, $\mathrm{n}$} & \multicolumn{2}{|c|}{ ATF2 expression, $n$} & \multirow[b]{2}{*}{ P-value } \\
\hline & & High & Low & \\
\hline Age, years & & & & 0.245 \\
\hline$<50$ & 49 & 30 & 19 & \\
\hline$\geq 50$ & 37 & 18 & 19 & \\
\hline Gender & & & & 0.891 \\
\hline Male & 55 & 31 & 24 & \\
\hline Female & 31 & 17 & 14 & \\
\hline Tumor size, $\mathrm{cm}$ & & & & $0.018^{\mathrm{a}}$ \\
\hline$<4$ & 34 & 16 & 18 & \\
\hline$\geq 4$ & 52 & 32 & 20 & \\
\hline TNM stage & & & & $0.002^{\mathrm{b}}$ \\
\hline $\mathrm{I}$ & 14 & 5 & 9 & \\
\hline II & 19 & 11 & 8 & \\
\hline III & 38 & 32 & 6 & \\
\hline IV & 15 & 9 & 6 & \\
\hline Vascular invasion & & & & 0.813 \\
\hline Yes & 51 & 29 & 22 & \\
\hline No & 35 & 19 & 16 & \\
\hline Metastasis & & & & $0.027^{\mathrm{a}}$ \\
\hline Yes & 52 & 34 & 18 & \\
\hline No & 34 & 14 & 20 & \\
\hline
\end{tabular}

${ }^{\mathrm{a}} \mathrm{P}<0.05,{ }^{\mathrm{b}} \mathrm{P}<0.01$. ATF2, activating transcription factor 2; TNM, tumor-node-metastasis.

a density of $2 \times 10^{3}$ cells/well in 96-well plates for attachment. MTT solution $(20 \mu \mathrm{l}, 5 \mathrm{mg} / \mathrm{ml})$ was added to each well at a specific time point $(12,24,48,72$ and $96 \mathrm{~h})$ and incubated for $4 \mathrm{~h}$ at $37^{\circ} \mathrm{C}$. Subsequently, the culture medium was removed and replaced with $150 \mu \mathrm{l}$ dimethyl sulfoxide to resolve the formazan. Absorbance was measured at $540 \mathrm{~nm}$ on a Model 550 microplate reader (Bio-Rad Laboratories, Hercules, CA, USA).

Statistical analysis. Statistical differences between groups were analyzed by performing the Student's t-test. The $\chi^{2}$ test was used to determine the difference in protein expression between tumor tissues and adjacent normal tissues. Survival rates were assessed using Kaplan-Meier survival curves (log-rank test). The Wilcoxon matched-pairs test was used to determine any significant difference in ATF2 mRNA expression between the normal and tumor tissues as well as reproducibility of the measurement of the expression of ATF2 and p-ATF2 in the 86 paraffin-embedded NSCLC tissues. The SPSS statistical software package, version 16.0 (SPSS, Inc., Chicago, IL, USA) was used in data processing and analyzing. Two-tailed $\mathrm{P}<0.05$ indicated a statistically significant difference.

\section{Results}

Expression of ATF2 in NSCLC cell lines and tissues. The expression of ATF2 in NSCLC cells was initially detected by western blotting and RT-qPCR. According to the results, the protein expression level of ATF2 was markedly higher in the H446, H1975, H1650, H1299 and PC9 cell lines (Fig. 1A). According to the western blot results, ATF2 is predominantly expressed in tumor tissues compared with non-tumor tissues (Fig. 1B). Furthermore, the mRNA expression level of ATF2 in 8/12 NSCLC tumor tissues was markedly higher than the adjacent normal tissues (Fig. 1C). In addition, ATF2 expression levels were significantly higher in tumor tissues compared to normal tissues according to the IHC results, as indicated in Fig. $1 \mathrm{D}(\mathrm{P}<0.01)$.

Association between ATF2/p-ATF2 expression and clinicopathological parameters. IHC was performed to assess the expression of ATF2 and p-ATF2 in 86 paraffin-embedded NSCLC tissues. The results showed that the expression of ATF2 was observed in the cytoplasm and nucleus of cancer cells, however, there was no difference in expression between the two locations (Fig. 2A). p-ATF2 was predominantly expressed in the nucleus of cancer cells compared with the cytoplasm $(\mathrm{P}<0.01$; Fig. $2 \mathrm{~B}$ and $\mathrm{C})$. In addition, the expression of p-ATF2 was detected in the cytoplasm. p-ATF2 was overexpressed in the majority of tumor tissues $(65 / 86$ cases, data not shown). The expression of ATF2 and p-ATF2 in adjacent non-cancerous tissue was also observed in certain cases (Fig. 2A and B). To determine the clinical significance by $\chi^{2}$ tests, the samples were grouped according to a low and high expression of ATF2 or p-ATF2. Table I shows a significant 
Table II. Correlation between p-ATF2 expression and clinicopathological parameters in non-small cell lung cancer.

\begin{tabular}{|c|c|c|c|c|}
\hline \multirow[b]{2}{*}{ Variable } & \multirow[b]{2}{*}{ Total cases, $\mathrm{n}$} & \multicolumn{2}{|c|}{ p-ATF2 expression, $n$} & \multirow[b]{2}{*}{ P-value } \\
\hline & & High & Low & \\
\hline Age, years & & & & 0.390 \\
\hline$<50$ & 49 & 36 & 13 & \\
\hline$\geq 50$ & 37 & 24 & 13 & \\
\hline Gender & & & & 0.502 \\
\hline Male & 55 & 37 & 18 & \\
\hline Female & 31 & 23 & 8 & \\
\hline Tumor size, cm & & & & 0.409 \\
\hline$<4$ & 34 & 22 & 12 & \\
\hline$\geq 4$ & 52 & 38 & 14 & \\
\hline TNM stage & & & & $0.040^{\mathrm{a}}$ \\
\hline I & 14 & 7 & 7 & \\
\hline II & 19 & 13 & 6 & \\
\hline III & 38 & 32 & 6 & \\
\hline Vascular invasion & & & & 0.781 \\
\hline Yes & 51 & 35 & 16 & \\
\hline No & 35 & 25 & 10 & \\
\hline Metastasis & & & & $0.015^{\mathrm{a}}$ \\
\hline Yes & 52 & 42 & 12 & \\
\hline No & 34 & 18 & 16 & \\
\hline
\end{tabular}

${ }^{\mathrm{a}} \mathrm{P}<0.05$. ATF2, activating transcription factor 2; TNM, tumor-node-metastasis.

A

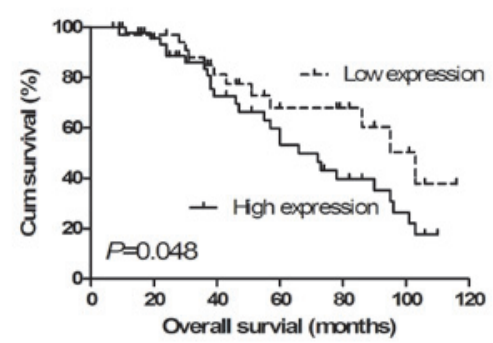

C

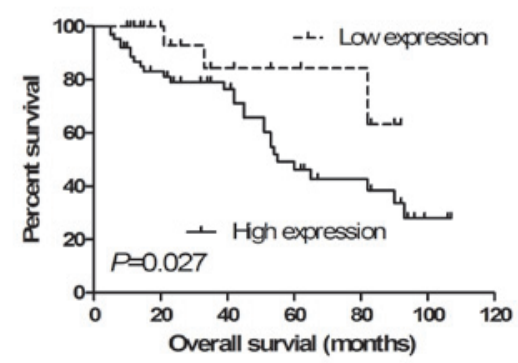

B

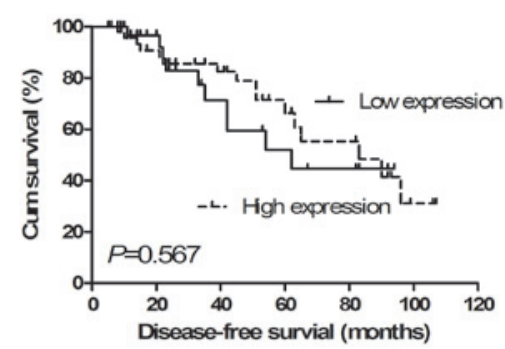

D

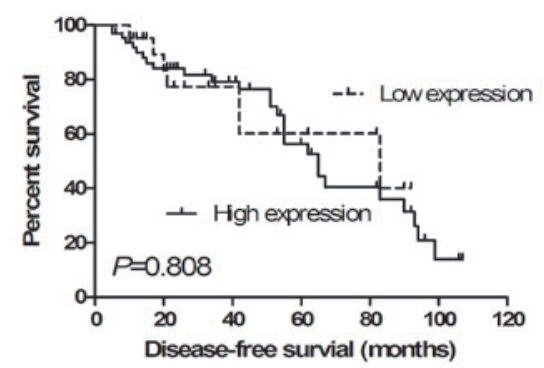

Figure 3. Association between activating transcription factor 2 (ATF2) expression and non-small cell lung cancer (NSCLC) prognosis. ATF2 protein expression level exhibited a prognostic role in (A) overall and (B) disease-free survival, as indicated by Kaplan-Meier analysis. (C and D) Prognostic role of phosphorylated-ATF2 in patients with NSCLC, demonstrated by (C) overall and (D) disease-free survival. Statistical significance was assessed using the log-rank test $(\mathrm{P}<0.05 ; \mathrm{n}=56)$.

difference between ATF2 expression and specific clinicopathological parameters was observed, including an advanced TNM stage $(\mathrm{P}=0.002)$, large tumor size $(\mathrm{P}=0.018)$ and metastasis
$(\mathrm{P}=0.027)$. However, there was no significant difference for the remaining clinicopathological parameters including age, gender or vascular invasion. For p-ATF2, patients with high 
A

H1299

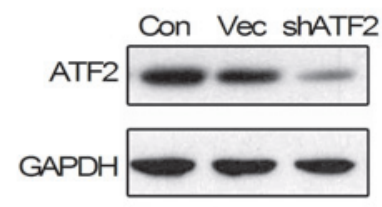

C

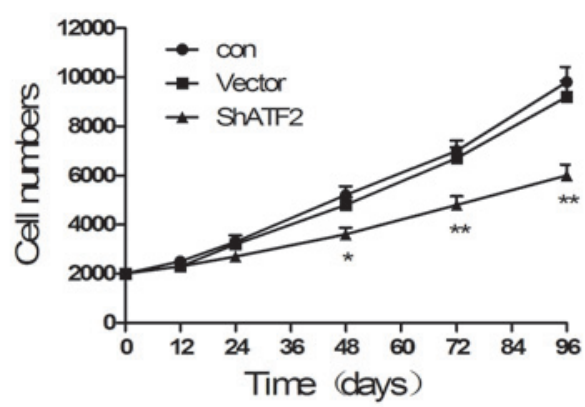

B

H1975

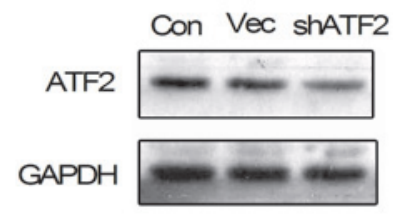

D

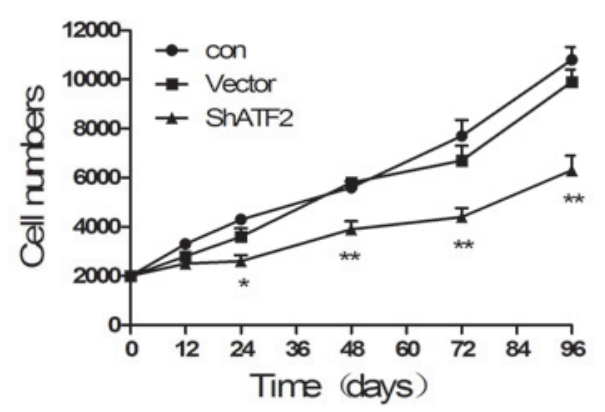

Figure 4. ATF2 shRNA markedly decreased cell proliferation in non-small cell lung cancer. (A and B) ATF2 shRNA noticeably downregulated the expression of ATF2 protein. Scramble and ATF2 shRNA were transfected into (A) H1299 and (B) H1975 cells for 24 h. Relative ATF2 expressions were detected by western blotting. (C) H1299 and (D) H1975 cell viabilities were determined using MTT assays. ${ }^{*} \mathrm{P}<0.05$ and ${ }^{* * *} \mathrm{P}<0.01$ vs. control. Data are presented as means \pm standard deviation of three independent experiments. Con, control; Vec, vector; shATF2, short hairpin activating transcription factor 2 .

p-ATF2 expression had a significant association with advanced TNM stage $(\mathrm{P}=0.040)$ and metastasis $(\mathrm{P}=0.015$; Table II $)$. The remaining clinicopathological parameters were not significantly associated with p-ATF2 expression ( $\mathrm{P}>0.05$; Table II).

Association between ATF2/p-ATF2 expression and prognosis in NSCLC. To determine the correlation between ATF2/ p-ATF2 expression and survival of patients with NSCLC, the Kaplan-Meier survival analysis was performed. As shown in Fig. 3A and B, the OS and disease-free survival (DFS) times in patients with a high level of ATF2 expression were significantly shorter than those of patients with a low level of ATF2 expression ( $\mathrm{P}<0.01$ and $\mathrm{P}<0.05$, respectively). For $\mathrm{p}-\mathrm{ATF} 2$, the results revealed that OS was significantly shorter in patients with a high p-ATF2 expression compared with patients with a low p-ATF2 expression $(\mathrm{P}<0.05)$; however, there was no significant difference in DFS ( $P>0.05$; Fig. $3 \mathrm{C}$ and D).

Downregulation of ATF2 inhibits cell proliferation. To determine the role of ATF2 in NSCLC cells, ATF2 knockdown cell models were generated in H1299 and H1975 cells. The ATF2 shRNA was proven to effectively downregulate the expression of ATF2 by western blotting (Fig. 4A and B). According to the results of the MTT assay, the knockdown of ATF2 in H1299 and H1975 cells led to a reduction in cell viability (Fig. $4 \mathrm{C}$ and $\mathrm{D} ; \mathrm{P}<0.05$ ). These data indicated that ATF2 may promote cell proliferation in NSCLC cells.

\section{Discussion}

ATF2, a member of the AP-1 family of transcription factors, is important in the regulation of cancer cell proliferation, invasion and survival by modulating various genes, such as c-Jun and c-Fos (9). A previous study revealed that ATF2 may play a potential role in breast cancer invasion and migration by targeting matrix metalloproteinase-2 (21). However, the role of ATF2 in NSCLC is poorly understood. To the best of our knowledge, the present study was the first to investigate the clinical role of ATF2 in Chinese patients with NSCLC. A marked increase in ATF2 expression was observed in NSCLC cancer tissues but not in corresponding normal tissues.

Notably, ATF2 has been demonstrated as a double-edged sword in different types of cancer. Using a ATF2-mutant K14-Cre mouse model, Bhoumik et al revealed that loss of ATF2 in keratinocytes could lead to elevated $\beta$-catenin expression and consequently result in skin tumor formation (22). Similarly, Maekawa et al identified that low expression of ATF2 in mammary tissue is more likely to form a tumor (23). By contrast, ATF2 gene transcriptional activity was activated in certain types of tumor. For example, overexpression of ATF2 was observed in patients with melanoma, and associated with poor outcome and metastasis (24). The expression of ATF2 was not proven to be an independent predictor of prognosis in patients with melanoma, however, the localization of ATF2 may still serve as a promising prognostic marker. Furthermore, Duffey et al demonstrated that when head and neck squamous cell carcinoma (HNSCC) cells were treated with tumor necrosis factor $\alpha$ and cisplatin, the expression of ATF2 in the nucleus was enhanced. Thus, enhanced ATF2 expression may predict a poor prognosis in patients with HNSCC (25). A previous report indicated that the dual functions of ATF2 may depend on its location: The cytosolic localization appeared to perform suppressor 
functions while the nuclear localization appeared to perform promoter functions (14). In addition, a previous study reported a correlation between ATF2 expression and clinicopathological parameters in breast cancer (26). Through immunohistochemical analysis of ATF2 and p-ATF2 protein expression levels, the present study identified important new information in this field. It was identified that high expression of ATF2 may be an indicator of poor prognosis for patients with NSCLC, as patients with high ATF2 expression exhibited advanced stage disease, larger tumors and metastasis. In addition, high expression of p-ATF2 was significantly associated with tumor stage and metastasis. These findings are consistent with a previous report, which identified that high ATF2 expression stimulated cell proliferation in human gastric cancer cells (27).

The present study investigated the association between ATF2 and p-ATF2 expression, and outcomes of patients with NSCLC. By performing Kaplan-Meier survival analysis, it was identified that high ATF2 expression in tumor tissues predicted significantly shorter OS and DFS, whereas p-ATF2 expression was only significantly associated with worse OS. A small number of previous reports have also demonstrated that ATF2 expression is associated with higher rates of metastasis and poor prognosis $(28,29)$. In addition, a previous study identified that ATF2 is involved in facilitating tumor progression and cell differentiation (8). Overall, it can be concluded that elevated ATF2 and p-ATF2 expression may be as a useful biological marker for predicting the outcomes of patients with NSCLC.

In summary, to the best of our knowledge, the present study is the first to provide clinical evidence that ATF2 and p-ATF2 may serve as promising predictors for NSCLC prognosis in a Chinese patient cohort. The results demonstrated that ATF2 expression is elevated in NSCLC tissues and associated with advanced clinical parameters, such as tumor size, metastasis and clinical stage. In addition, p-ATF2 overexpression was detected in tumor tissues and indicated shorter survival of patients with NSCLC. The results of the current study suggest that ATF2 and p-ATF2 may be useful prognostic biomarkers for patients with NSCLC. Thus, the potential mechanisms of ATF2 leading to poor prognosis should be further analyzed and the current results should be confirmed in other NSCLC cohorts.

\section{Acknowledgements}

The current study was supported by the Educational Commission of Jiangxi Province of China (no. 20143056).

\section{References}

1. Jemal A, Bray F, Center MM, Ferlay J, Ward E and Forman D: Global cancer statistics. CA Cancer J Clin 61: 69-90, 2011.

2. D'Addario $\mathrm{G}$ and Felip E; ESMO guidelines working group: Non-small-cell lung cancer: ESMO clinical recommendations for diagnosis, treatment and follow-up. Ann Oncol 4 (Suppl 20): S68-S70, 2009.

3. McElnay P and Lim E: Adjuvant or neoadjuvant chemotherapy for NSCLC. J Thorac Dis 6 (Suppl 2): S224-S227, 2014.

4. Ellis PM, Coakley N, Feld R, Kuruvilla S and Ung YC: Use of the epidermal growth factor receptor inhibitors gefitinib, erlotinib, afatinib, dacomitinib, and icotinib in the treatment of non-small-cell lung cancer: A systematic review. Curr Oncol 22: e183-e215, 2015
5. NSCLC Meta-analyses collaborative group; Arriagada R, Auperin A, et al: Adjuvant chemotherapy, with or without postoperative radiotherapy, in operable non-small-cell lung cancer: Two meta-analyses of individual patient data. Lancet 375: 1267-1277, 2010

6. Spiro SG, Tanner NT, Silvestri GA, et al: Lung cancer: Progress in diagnosis, staging and therapy. Respirology 15: 44-50, 2010.

7. Lee IT, Lin CC, Cheng SE, Hsiao LD, Hsiao YC and Yang CM: TNF- $\alpha$ induces cytosolic phospholipase A2 expression in human lung epithelial cells via JNK1/2- and p38 MAPK-dependent AP-1 activation. PloS One 8: e72783, 2013.

8. Bengoa-Vergniory N, Gorroño-Etxebarria I, González Salazar I and Kypta RM: A switch from canonical to noncanonical Wnt signaling mediates early differentiation of human neural stem cells. Stem Cells 32: 3196-3208, 2014.

9. Lopez-Bergami P, Lau E and Ronai Z: Emerging roles of ATF2 and the dynamic AP1 network in cancer. Nat Rev Cancer 10: 65-76, 2010.

10. Bhoumik A, Lopez-Bergami P and Ronai Z: ATF2 on the double-activating transcription factor and DNA damage response protein. Pigment Cell Res 20: 498-506, 2007.

11. Lau E and Ronai ZA: ATF2-at the crossroad of nuclear and cytosolic functions. J Cell Sci 125: 2815-2824, 2012.

12. Shah M, Bhoumik A, Goel V, et al: A role for ATF2 in regulating MITF and melanoma development. PLoS Genet 6: e1001258, 2010.

13. Lau $\mathrm{E}$, Kluger $\mathrm{H}$, Varsano $\mathrm{T}$, et al: $\mathrm{PKC} \varepsilon$ promotes oncogenic functions of ATF2 in the nucleus while blocking its apoptotic function at mitochondria. Cell 148: 543-555, 2012.

14. Varsano T, Lau E, Feng Y, et al: Inhibition of melanoma growth by small molecules that promote the mitochondrial localization of ATF2. Clin Cancer Res 19: 2710-2722, 2013.

15. An JJ, Shi KJ, Wei W, et al: The ROS/JNK/ATF2 pathway mediates selenite-induced leukemia NB4 cell cycle arrest and apoptosis in vitro and in vivo. Cell Death Dis 4: e973, 2013.

16. Laskin WB and Miettinen M: Epithelioid sarcoma: New insights based on an extended immunohistochemical analysis. Arch Pathol Lab Med 127: 1161-1168, 2003.

17. Song H, Ki SH, Kim SG and Moon A: Activating transcription factor 2 mediates matrix metalloproteinase-2 transcriptional activation induced by p38 in breast epithelial cells. Cancer Res 66: 10487-10496, 2006.

18. Knippen S,Löning T, Müller V, et al: Expression and prognostic value of activating transcription factor 2 (ATF2) and its phosphorylated form in mammary carcinomas. Anticancer Res 29: 183-189, 2009.

19. Schmittgen TD and Livak KJ: Analyzing real-time PCR data by the comparative C (T) method. Nat Protoc 3: 1101-1108, 2008.

20. Yamada $\mathrm{H}$ and Sano Y: The biotinylation of the rabbit serotonin antibody and its application to immunohistochemical studies using the two-step ABC method. Histochemistry 83: 285-289, 1985.

21. Zhao Y, Li Y, Han J, et al: Helicobacter pylori enhances CIP2A expression and cell proliferation via JNK2/ATF2 signaling in human gastric cancer cells. Int J Mol Med 33: 703-710, 2014.

22. Bhoumik A, Huang TG, Ivanov V, et al: An ATF2-derived peptide sensitizes melanomas to apoptosis and inhibits their growth and metastasis. J Clin Invest 110: 643-650, 2002.

23. Maekawa T, Shinagawa T, Sano Y, et al: Reduced levels of ATF-2 predispose mice to mammary tumors. Mol Cell Biol 27: 1730-1744, 2007.

24. Berger AJ, Kluger HM, Li N, et al: Subcellular localization of activating transcription factor 2 in melanoma specimens predicts patient survival. Cancer Res 63: 8103-8107, 2003

25. Duffey D, Dolgilevich S, Razzouk S, Li L, Green R and Gorti GK: Activating transcription factor-2 in survival mechanisms in head and neck carcinoma cells. Head Neck 33: 1586-1599, 2011.

26. Knippen S, Löning T, Müller V, et al: Expression and prognostic value of activating transcription factor 2 (ATF2) and its phosphorylated form in mammary carcinomas. Anticancer Res 29: 183-189, 2009.

27. Zhao Y, Li Y, Han J, et al: Helicobacter pylori enhances CIP2A expression and cell proliferation via JNK2/ATF2 signaling in human gastric cancer cells. Int J Mol Med 33: 703-710, 2014.

28. Bhoumik A, Huang TG, Ivanov V, et al: An ATF2-derived peptide sensitizes melanomas to apoptosis and inhibits their growth and metastasis. J Clin Invest 110: 643-650, 2002.

29. Deng X, Liu H, Huang J, et al: Ionizing radiation induces prostate cancer neuroendocrine differentiation through interplay of CREB and ATF2: Implications for disease progression. Cancer Res 68: 9663-9670, 2008. 